\title{
Research of error detection and compensation of CNC machine tools based on laser interferometer
}

\author{
Yueming Zhang ${ }^{1, a}$, Xunxun $\mathrm{Chu}^{2, \mathrm{~b}}$ and Shenchun Yang ${ }^{2, \mathrm{c}}$ \\ ${ }^{1}$ Beijing University of Technology, Beijing 100124, China; \\ ${ }^{2}$ Beijing University of Technology, Beijing 100124, China; \\ azhangym@bjut.edu.cn, bchuxx@emails.bjut.edu.cn, cyangshenchun@emails.biut.edu.cn
}

Keywords: laser interferometer, positioning accuracy, error compensation

\begin{abstract}
Taking a certain type of precision ultra-precision $\mathrm{CNC}$ grinding machine as an experimental platform, the research uses Renishaw XL-80 laser interferometer in testing the machine tools positional accuracy and repeat positioning accuracy in the before and after position of error compensation. Through the establishment of deviation mathematical model with VDI standard, the paper uses the Matlab powerful data analysis functions to calculate machine movement error value and draws the error distribution curve. Using the error compensation mechanism of FANUC system for pitch compensation and backlash compensation, so that the machine tools meet the requirements of precision ultra-precision machining accuracy.
\end{abstract}

\section{Introduction}

With the rapid development of China's industrial 4.0, precision ultra-precision machining technology has become an important direction of the transformation of China's machinery industry. Precision ultra-precision machining is guaranteed by the accuracy of CNC machine tools. At the same time, it also put forward higher requirements for the development and application of domestic precision ultra-precision CNC machine tools. Positioning accuracy is an important indicator of the precision of ultra-precision $\mathrm{CNC}$ machine, and the ball screw pitch error and the ball screw backlash error are important indicators to ensure the positioning accuracy of CNC machine tools. Although the level of precision of ball screw is higher and higher, in the long-term use of machine tools, due to the wear and tear of the functional components, its accuracy will be a significant decline. Therefore, we need to regularly test the positioning accuracy of machine tools to ensure the machining accuracy of the workpiece.

This paper, according to the position accuracy problem of a model of precision ultra-precision CNC grinding machine, describes the principle, installation and error compensation mechanism of the Renishaw XL-80 dual-frequency laser interferometer. This paper uses the Matlab analysis functions to calculate the errors. This article has detected and compensated the machine pitch error and backlash error to achieve the positioning accuracy by the $9.1 \mu \mathrm{mto} 2.7 \mu \mathrm{m}$.

\section{XL-80 dual-frequency laser interferometer}

Introduction of laser interferometer. XL-80 laser interferometer produced by Renishaw is a high precision instrument of linear machine error measurement and calibration. It has high measuring accuracy, good portability and error compensation function etc, so it is widely used in precision measurement and calibration.XL-80 laser head performance indicators are shown in Table 1. 
Table 1 XL-80 Laser head performance indicators

\begin{tabular}{l|c}
\hline Index items & Parameter \\
\hline System accuracy $(\mathrm{ppm})$ & \pm 0.5 \\
Laser precision $(\mathrm{ppm})$ & 0.05 \\
Resolution $(\mu \mathrm{m})$ & 0.001 \\
Maximum measurement speed $(\mathrm{m} / \mathrm{min})$ & 2404 \\
Maximum sampling frequency $(\mathrm{KHz})$ & 50 \\
Measuring range(m) & $0-80$ \\
Warm-up time $(\mathrm{min})$ & $\sim 5$ \\
\hline
\end{tabular}

Measuring principle of laser interferometer. Renishaw XL-80 laser interferometer emitted sine wave wavelength of $633 \mathrm{~nm}$. The laser has the following characteristics: wavelength is precisely known, long and short of the wave is consistent with the phase of the wave. After the laser light emitted from the laser head, it is divided into two beams by spectroscope(A). One way laser is reflected to the fixed mirror(B) and another way laser hits the moving mirror(C). After reflection, the measuring beam is formed. As shown in Fig. 1.

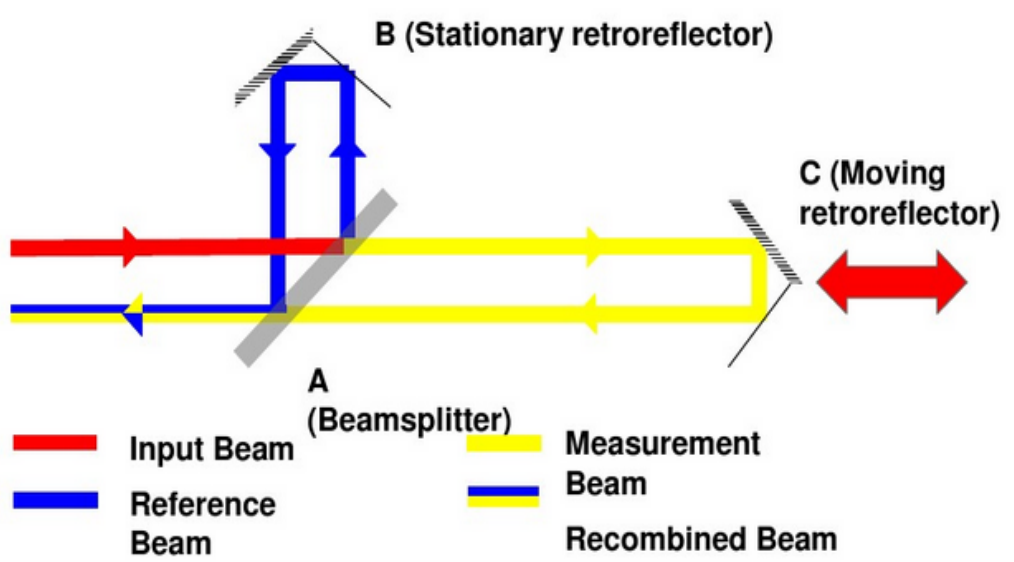

Fig. 1 The principle diagram of laser interferometer measurement

Measuring beam and reference beam interfere with each other after through a spectroscope again. If the two coherent beams in the same phase, then let the two coherent beams peaks superimpose to form bright stripes. If the two coherent beams in the opposite phase, then let the two coherent beams peaks and troughs superimpose to form dark stripes.As shown in Fig. 2.

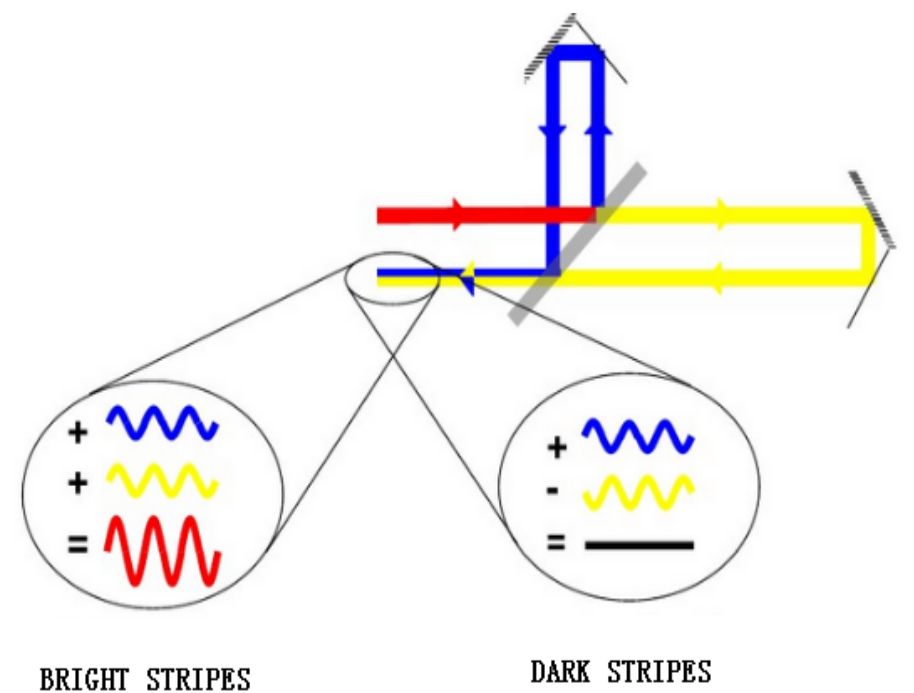

Fig. 2 Measuring beam and reference beam

These stripes are counted out to obtain doppler difference of $\operatorname{arrival}(\Delta \mathrm{f})$, so it can calculate path difference of the two coherent beams. The calculations are as follows:

$$
N=\int_{0}^{t} \Delta f d t
$$


$\Delta f=2(v / c) f$

$v=d l / d t$

$f=c / \lambda$

$N=\int_{0}^{t} \Delta f d t=(2 / \lambda) \int_{0}^{t} d L=2 L / \lambda$

$L=(\lambda / 2) N$

In the formula:

$\mathrm{N}$ : the cumulative number of pulses
$\lambda:$ a laser wavelength
$c:$ the speed of light

\section{The mathematical model of error}

According to the principle of mathematical statistics: the paper uses the mean and standard deviation (SD) to analyze the monitoring data. In the following formula, $\mathrm{i}(\mathrm{i}=1 \sim \mathrm{m})$ is the measuring target location, $\mathrm{j}(\mathrm{j}=1 \sim \mathrm{n})$ is the Number of measurement, $\uparrow$ represents the forward movement, $\downarrow$ represents the backward movement.

The average position deviation: $\overline{X_{i}}$

$$
\begin{aligned}
& X_{i j} \uparrow=P_{i j} \uparrow-P_{i} \\
& X_{i j} \downarrow=P_{i j} \downarrow-P_{i} \\
& \bar{X}_{i} \uparrow=\frac{1}{n} \sum_{j=1}^{n} X_{i j} \uparrow \\
& \bar{X}_{i} \downarrow=\frac{1}{n} \sum_{j=1}^{n} X_{i j} \downarrow \\
& \bar{X}_{i}=\frac{X_{i} \uparrow+X_{i} \downarrow}{2}
\end{aligned}
$$

The average standard deviation: $\overline{S_{i}}$

$$
S_{i} \uparrow=\sqrt{\frac{1}{n-1} \sum_{j=1}^{n}\left(X_{i j} \uparrow-\overline{X_{i}} \uparrow\right)^{2}}
$$

$S_{i} \downarrow=\sqrt{\frac{1}{n-1} \sum_{j=1}^{n}\left(X_{i j} \downarrow-\overline{X_{i}} \downarrow\right)^{2}}$

$\bar{S}_{i}=\frac{S_{i} \uparrow+S_{i} \downarrow}{2}$

The average position dispersion: $\overline{P_{s i}}$

$P_{s i}=6 \overline{S_{i}}$

$\overline{P_{s i}}=\frac{1}{n} \sum_{j=1}^{n} P_{s i}$

The average reverse difference: $\bar{U}$

$$
\begin{aligned}
& \overline{U_{i}}=\overline{X_{i}} \uparrow-\overline{X_{i}} \downarrow \\
& U_{i}=\left|\overline{X_{i}} \uparrow-\overline{X_{i}} \downarrow\right| \\
& U=\max \left(\left|\overline{X_{i}} \uparrow-\overline{X_{i}}\right|\right)
\end{aligned}
$$


$\bar{U}=\frac{1}{n} \sum_{j=1}^{n} \overline{U_{i}}$

Positioning accuracy: $P$

$P=\max \left(\overline{X_{i}}+\frac{3\left(S_{i} \uparrow+S_{i} \downarrow\right)}{2}+\frac{U_{i}}{2}\right)-\min \left(\overline{X_{i}}-\frac{3\left(S_{i} \uparrow+S_{i} \downarrow\right)}{2}-\frac{U_{i}}{2}\right)$

Repeat positioning accuracy: $R$

$R=\max \left(P_{s i}\right)$

Positional deviation: $M$

$M=\max \left(\overline{X_{i}}\right)-\min \left(\overline{X_{i}}\right)$

\section{Data analysis and error correction}

Incremental compensation is an error compensation method, which takes the adjacent error difference between two points as compensation basis. This paper takes the machine $\mathrm{Z}$ axis as experimental subject. Through analysis of the data, it uses the incremental method for error compensation.After a series of data compensation, the precision of the machine tool of the $\mathrm{Z}$ axis is greatly improved. The $\mathrm{X}$ axis and $\mathrm{Y}$ axis can use the same method for error compensation. The results are shown in Fig. 3 and Fig. 4.

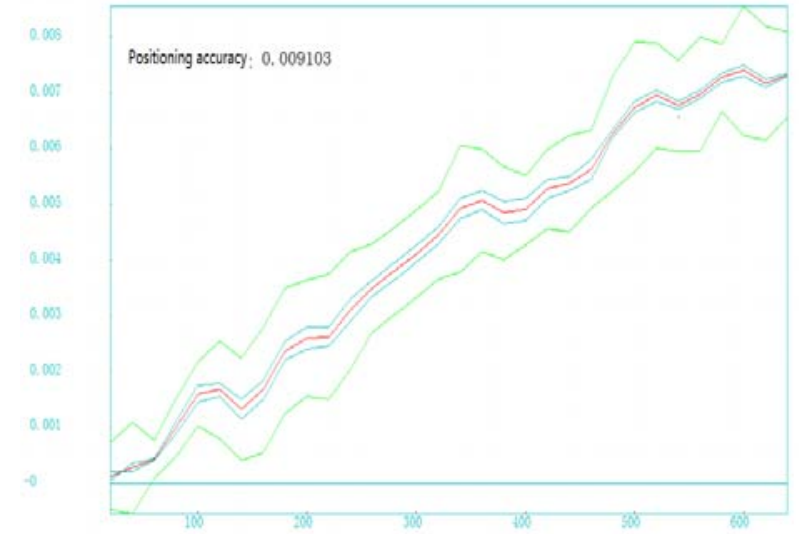

Fig. 3 Precision before compensation

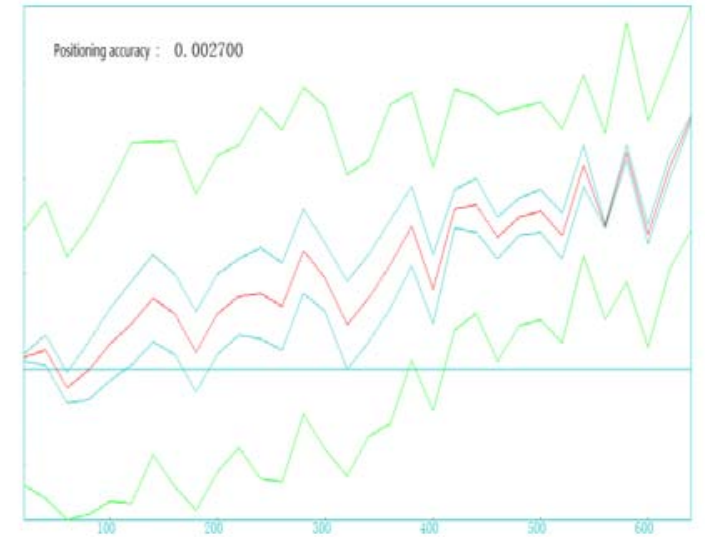

Fig. 4 Precision after compensation

\section{Summary}

This article uses the laser interferometer to detect and compensate a machine screw pitch error compensation. Machine accuracy is improved greatly. This article achieve the positioning accuracy by the $9.1 \mu \mathrm{mto} 2.7 \mu \mathrm{m}$.

Practice and data show that periodic precision detection and error proofing are very important to ensure accuracy of machine tools. Through the pitch error compensation, the parts processing quality can be improved.

\section{Acknowledgments}

This work was financially supported by "the National Science and Technology Major Project(2012ZX04002051)" and "the Beijing Municipal Education Commission Science and Technology Innovation Platform(PXM2012-014204-00-00168)”.

\section{References}

[1] W. Lei, Y. Hsu, Error measyrment of five-axis CNC machines with 3D probe-ball, Journal of Materials Processing Technology, 139(2003)127-133. 
[2] A.C. Okafor, Y.M. Ertekin, Derivation of machine tool error models and error compensation procedurefor three axes vertical machining center using rigid body kinematics, International Journal of Machine Tools \& Manufacture, 40(2000)1120-1221.

[3] A.C. Okafor, Y.M. Ertekin, Derivation of machine tool error models and error compensation procedurefor three axes vertical machining center using rigid body kinematics, International Journal of Machine Tools, 105(2000)410-419. 